\title{
A depression management programme reduced depression in frequent users of health care but did not reduce healthcare visits
}

\author{
Katzelnick DJ, Simon GE, Pearson SD, et al. Randomized trial of a depression management program in high utilizers of \\ medical care. Arch Fam Med 2000 Apr;9:345-51.

\section{QUESTION: Does a programme to identify and treat depression in frequent users of medical care improve clinical outcomes and reduce total healthcare use?}

Design

Cluster randomised \{allocation concealed*\}†, blinded (telephone assessment), ${ }^{*}$ controlled trial with 1 year follow up.

\section{Setting}

Primary care clinics of 3 health management organisations (HMOs) in the US (Wisconsin, Washington, and Massachusetts).

Source of funding:

Inc.

For correspondence:

Dr D J Katzelnick,

Madison Institute of

Medicine, 7617

Mineral Point Road,

Madison, WI 53562

USA.Fax +1 608827

2444

Abstract and

commentary also

appear in

Evidence-Based Mental

Health.
Pfizer Pharmaceuticals

\section{Patients}

407 patients who were 25-63 years of age (mean age $45 \mathrm{y}$, $77 \%$ women), had continuous health plan enrolment for $\geqslant 2$ years, were frequent healthcare users (ambulatory visits per year above the 85 th centile for previous 2 years), and screened positive for current major depression or major depression in partial remission with a score $\geqslant 15$ on the Hamilton Depression Rating Scale (HDRS). Exclusion criteria included recent treatment for substance abuse, previous treatment for schizophrenia or bipolar disorder, life threatening medical illness, or active treatment for depression by a mental health specialist. $93 \%$ of patients completed the blinded telephone assessment at 12 months.

A depression management programme (DMP) v usual care for frequent healthcare users $\$$

\begin{tabular}{|c|c|c|c|}
\hline \multirow[b]{2}{*}{ Outcomes } & \multicolumn{2}{|c|}{ Mean score decrease } & \multirow{2}{*}{$\begin{array}{l}\text { Difference in mean } \\
\text { score decrease }\end{array}$} \\
\hline & DMP & Usual care & \\
\hline HDRS score at 6 weeks & 3.3 & 2.0 & 1.3 \\
\hline HDRS score at 3 months & 5.6 & 3.9 & 1.7 \\
\hline HDRS score at 6 months & 7.3 & 4.0 & 3.3 \\
\hline HDRS score at 12 months & 9.2 & 5.6 & 3.6 \\
\hline
\end{tabular}

fHDRS $=$ Hamilton Depression Rating Scale. Scores corrected for intracluster correlation among patients of the same physician. All differences are statistically significant.

\section{Intervention}

82 physician practices were allocated to the depression management programme (DMP) $(\mathrm{n}=218)$, and 81 were allocated to usual care $(n=189)$. DMP consisted of physician education (2 hour training session and psychiatrist consultants at each HMO), patient education (a booklet entitled Depression Isn't Just a Medical Problem and videotaped educational materials), antidepressant treatment (a pharmacotherapy algorithm with adjustment as needed), and treatment coordination.

\section{Main outcome measures}

Change in scores on the HDRS and number of healthcare visits.

\section{Main results}

Analysis was by intention to treat. Patients in the DMP group had greater improvements in score at 6 weeks $(\mathrm{p}=0.04), 3$ months $(\mathrm{p}=0.02), 6$ months $(\mathrm{p}<0.001)$, and 12 months $(\mathrm{p}<0.001)$ than patients in the usual care group (table). Patients in the DMP group had more healthcare visits (mean increase 1.6 visits) during follow up than they had had the previous year, whereas patients in the usual care group had a decrease in healthcare visits (mean decrease 2.0 visits) $(p=0.02$ for the difference between groups).

\section{Conclusion}

In depressed patients who are frequent users of general medical care, a depression management programme led to greater clinical improvement but also to increased healthcare visits.

\section{*See glossary.}

$\dagger$ Information provided by author.

\section{COMMENTARY}

Depressive illness is common and disabling and is mostly managed in primary care without recourse to specialist services. Received wisdom is that management is suboptimal: up to $50 \%$ of depressive conditions are missed by practitioners, and treatment of diagnosed patients is of inadequate intensity and duration. Educational interventions for practitioners that use clinical guidelines to improve recognition and management have been evaluated, but they have shown no benefit.

At least 3 hypotheses may explain this failure: education may be insufficient to change practitioner behaviour, study design may have been inadequate to detect true benefit, or the principles on which guidelines are based may be at fault. The last reason is likely because most of the evidence base comes from secondary care, reflecting a lack of research in primary care. "Sensitivity" of practitioners has been emphasised at the expense of "specificity," which may result in a failure to target patients who would benefit most from more intensive management.

The studies by Katzelnick $e t$ al and Simon $e t$ al report the efficacy and cost effectiveness of a practice based intervention to improve depression management in the managed care context of 3 US HMOs. In a 2 stage screening procedure, patients with consultation rates above the 85th centile for $\geqslant 2$ years were identified, and those in whom evidence existed of untreated depressive disorder (Diagnostic and Statistical Manual, Fourth Edition, diagnosis and HDRS score $\geqslant 15$ ) were recruited (about $5 \%$ of the total registered population). Half the sample had $\geqslant 1$ comorbid chronic physical illness, presumably accounting in part for their high consultation rates. The study had high completion rates, but only patients enrolled with the HMO for $\geqslant 2$ years were eligible. This study characteristic limits the generalisability of the work to other settings (such as general practice) and biases the results against such groups as the long term unemployed or the elderly.

DMPs have already been shown to be of benefit to unselected depressed patients in primary care. In this study, patients in the DMP had better outcomes than those receiving usual care, with significantly greater reductions in depression scores and higher quality of life ratings. The authors estimate a number 


\section{A depression management programme increased depression free days and costs in depressed frequent users of general health care}

Simon GE, Manning WG, Katzelnick DJ, et al. Cost-effectiveness of systematic depression treatment for high utilizers of general medical care. Arch Gen Psychiatry 2001 Feb;58:181-7.

\section{QUESTION: In depressed patients who are frequent healthcare users, what is the incremental cost effectiveness of a depression management programme (DMP)?}

\section{Design}

Cost effectiveness analysis of a cluster randomised \{allocation concealed $* \dagger$, partially blinded (telephone assessment),* controlled trial with 12 months follow up.

\section{Setting}

3 health maintenance organisations (HMOs) in the US.

\section{Patients}

407 patients (mean age 45 y, $77 \%$ women) who were frequent users of general medical care $(>85$ th centile for the number of outpatient visits in each of the previous 2 y) and were depressed (Hamilton Depression Rating Scale [HDRS] score $\geqslant 15$ ). Exclusion criteria included active treatment for depression in the previous 90 days or contraindications to depression treatment. Analyses included $92 \%$ of patients for healthcare use and $91 \%$ for cost effectiveness.

\section{Intervention}

$\{82\} \neq$ physician practices were allocated to a DMP $(\mathrm{n}=218)$, and $\{81\}$ were allocated to usual care $(\mathrm{n}=189)$. DMP consisted of patient and physician education and telephone care management, antidepressant treatment for most patients, and psychiatric consultation for non-responders.

\section{Main cost and outcome measures}

The main outcome was number of depression free days (estimated by interpolation). Direct costs were assessed for all services provided or paid for by health plans in
1996 US dollars. Costs for time in treatment were estimated as lost wages. Results were adjusted for age, sex, study site, baseline measures of depression severity and health status, and clustering of patients by physicians.

\section{Main results}

The DMP group had more depression free days than the usual care group (229.3 v $181.9 \mathrm{~d}$; mean adjusted difference $47.4 \mathrm{~d}, 95 \%$ CI 26.6 to $68.2 \mathrm{~d}$ ). The table shows the incremental costs of the DMP relative to usual care.

\section{Conclusions}

In depressed patients who are frequent users of general health care, a depression management programme was effective for improving clinical outcomes at increased health services cost. Outpatient and inpatient services each cost approximately $\$ 20$ per additional depression free day.

*See glossary.

†Information provided by author.

$\ddagger$ Katzelnick DJ, Simon GE, Pearson SD, et al. Arch Fam Med 2000;9:345-51.

Incremental cost of a depression management programme relative to usual care

\begin{tabular}{|c|c|c|}
\hline Outcomes at 12 months & $\begin{array}{l}\text { Adjusted incremental } \\
\text { cost }(95 \% \mathrm{Cl}) \S\end{array}$ & $\begin{array}{l}\text { Adjusted cost per additional } \\
\text { depression free day }(\mathrm{Cl}) \S\end{array}$ \\
\hline Outpatient health services & $\$ 1008$ (534 to 1383 ) & $\$ 21.12$ (10.53 to 37.61$)$ \\
\hline Outpatient plus inpatient services & $\$ 1974$ (848 to 3171) & $\$ 41.34$ (16.04 to 81.03$)$ \\
\hline $\begin{array}{l}\text { Outpatient and inpatient services } \\
\text { plus time in treatment costs }\end{array}$ & $\$ 2475$ (880 to 4138 ) & $\$ 51.84$ (17.37 to 108.47$)$ \\
\hline
\end{tabular}
ion.

\section{COMMENTARY-continued from previous page}

needed to treat of 5 to achieve 1 additional remission, although it is not clear how remission was defined for this calculation. The 2 studies were screening studies, however, and the "number needed to screen" to achieve 1 additional remission is close to 17. Consultation rates in the intervention group increased, whereas those in the usual care group decreased slightly.

It is not possible to identify which component of the programme was most beneficial, but it seems probable on clinical grounds that the initial visit for assessment and initiation of management (which did not occur in the usual care group) would have had considerable effect.

In analysing the cost effectiveness of the programme, the construct of "depression free days" was used. It is important to understand how these days were derived. As depression scores were only assessed at 6 weeks and 3, 6, and 12 months, most of the data were interpolated. HDRS scores $\leqslant 7$ were taken as "depression free" (score 0 ), whereas scores $\geqslant 22$ were taken as "fully symptomatic" (score 1). Linear interpolation was used to model recovery, allowing calculation of a number between 0 and 1 for each day; "depression free days" were then calculated by dividing the total scores by the number of days in the period between estimates. This construct is clearly notional, and its name is misleading because many of the periods contained no true depression free days, only partial depression free days.

The costs of the intervention appear high, and no evidence existed of the hoped for "cost offset" effect by reduction in other sources of healthcare costs, perhaps not surprising given the high prevalence of comorbid physical illness. The authors observe that the study was insufficiently powered to compare frequency of inpatient admission, which is costly; this lack might have led to failure to detect benefits. The study also had only a 1 year follow up, and benefits may take longer to be detected.

The clinical bottom line is that it is possible to identify unmet needs and improve outcomes in this segment of the primary care population, but substantial additional resources are required. Although these are not out of line with the costs of treating other important conditions, priorities have to be established to permit shifting of existing resources away from other therapeutic areas or investment of greater resources in this one.

Robert Peveler, MA, DPhil, BM, BCh University of Southampton and Royal South Hants Hospital Southampton, UK 